\title{
Rydberg-Resolved Resonant Inelastic Soft X-Ray Scattering: Dynamics at Core Ionization Thresholds
}

\author{
J.-E. Rubensson, ${ }^{1}$ J. Söderström, ${ }^{1}$ C. Binggeli, ${ }^{1}$ J. Gråsjö, ${ }^{2}$ J. Andersson, ${ }^{1,3}$ C. Såthe, ${ }^{4}$ F. Hennies, ${ }^{4}$ V. Bisogni, ${ }^{5}$ Y. Huang,${ }^{5}$ \\ P. Olalde, ${ }^{5}$ T. Schmitt, ${ }^{5}$ V. N. Strocov, ${ }^{5}$ A. Föhlisch, ${ }^{6,7}$ B. Kennedy, ${ }^{6}$ and A. Pietzsch ${ }^{6}$ \\ ${ }^{1}$ Department of Physics and Astronomy, Uppsala University, Box 516, S-751 20 Uppsala, Sweden \\ ${ }^{2}$ Department of Pharmacy, Uppsala University, Box 580, SE-751 23 Uppsala, Sweden \\ ${ }^{3}$ Centre for Quantum Technologies, National University of Singapore, 3 Science Drive 2, Singapore 117543, Singapore \\ ${ }^{4}$ MAX IV Laboratory, Lund University, Box 118, 22100 Lund, Sweden \\ ${ }^{5}$ Swiss Light Source, Paul Scherrer Institut, CH-5232 Villigen PSI, Switzerland \\ ${ }^{6}$ Institute for Methods and Instrumentation in Synchrotron Radiation Research G-ISRR, \\ Helmholtz-Zentrum Berlin für Materialien und Energie Albert-Einstein-Strasse 15, 12489 Berlin, Germany \\ ${ }^{7}$ Institut für Physik und Astronomie, Universität Potsdam, Karl-Liebknecht-Strasse 24-25, 14476 Potsdam, Germany
}

(Received 30 November 2014; published 31 March 2015)

\begin{abstract}
Resonant inelastic $\mathrm{x}$-ray scattering spectra excited in the immediate vicinity of the core-level ionization thresholds of $\mathrm{N}_{2}$ have been recorded. Final states of well-resolved symmetry-selected Rydberg series converging to valence-level ionization thresholds with vibrational excitations are observed. The results are well described by a quasi-two-step model which assumes that the excited electron is unaffected by the radiative decay. This threshold dynamics simplifies the interpretation of resonant inelastic x-ray scattering spectra considerably and facilitates characterization of low-energy excited final states in molecular systems.
\end{abstract}

DOI: 10.1103/PhysRevLett.114.133001

The high brilliance of new x-ray sources promises realization of the full potential of resonant inelastic $\mathrm{X}$-ray scattering (RIXS) as a technique for investigation of materials and processes in fields ranging from fundamental atomic and molecular physics to materials science and biology [1-3]. Here, we address the RIXS phenomenology for excitation in the immediate vicinity of core ionization thresholds by analyzing the population of Rydberg states in the $\mathrm{N}_{2}$ molecule.

The similarities between RIXS and well-established resonant photoemission (RPE) are often emphasized. In a two-step picture, the two processes both start by photoexcitation of a core hole state. Only, in the second step, they differ as RIXS proceeds by spontaneous emission of a photon when the core hole is filled by an outer electron, while RPE proceeds by emission of an electron in an autoionization process. Also, in more rigorous scattering theory, both processes are often described using the same KramersHeisenberg formalism, and they are often termed radiative (RIXS) and nonradiative (RPE) X-ray scattering [4].

One significant difference between the two processes concerns dynamics in the immediate vicinity of ionization thresholds. Assume that the same intermediate state with a core hole and one electron in a Rydberg orbital or in the continuum is excited. The decay via electron emission changes the charge state and has a large influence on the remaining electrons. The initially excited electron is strongly affected and a wide distribution of ionic Rydberg of continuum final states are populated. It has been
PACS numbers: $33.20 . \mathrm{Rm}, 32.80 . \mathrm{Aa}, 33.20 . \mathrm{Fb}, 33.80 . \mathrm{Rv}$

investigated how shake processes at below-threshold excitation develop into postcollision interaction (PCI) as the excitation energy is varied over core ionization thresholds [5]. If decay by photon emission constitutes the second step, the change in potential is much smaller, and consequently, shake and PCI processes are much less pronounced. Although radiative electron rearrangement and radiative Auger have been reported [6,7], any counterpart in the radiative channel to shake processes and PCI at threshold is yet to be observed [8-10]. In the complete absence of such effects, the interpretation of the RIXS spectra becomes particularly simple. Then, the electron excited in the first step remains as a pure spectator during the radiative decay, and the distribution of Rydberg or continuum states excited in the first step of the process will determine the population of final Rydberg states. No interference between different scattering channels would occur since each intermediate state reaches a specific final state [11].

Here, we present RIXS spectra of gas-phase $\mathrm{N}_{2}$ excited close to the core-level ionization thresholds. We find a rich phenomenology as final Rydberg states with both valence and vibrational excitations are populated. Assignment is facilitated by the dramatic excitation-energy dependence of the distribution of final Rydberg states. A simple two-step model, which assumes that the electron excited in the first step is unaffected by the radiative decay, describes the experimental results well. Using the symmetry selection rules, a multitude of Rydberg states can be assigned in the 
nitrogen molecule, and it is suggested that this approach can be used in more complex molecular systems, and in experiments where RIXS data are used to construct "absorption spectra with removed lifetime broadening" [12].

The experiment was performed at the ADRESS beam line [13] of the Swiss Light Source, Paul Scherrer Institut Villigen, using the SAXES spectrometer [14], measuring at $90^{\circ}$ scattering angle. The overall energy resolution was around $50 \mathrm{meV}$, allowing for separation of individual vibrations [15] and Rydberg states up to $n \approx 8-9$. Linearly polarized incident radiation with the polarization vector parallel to the scattering plane was used, and gas-phase measurements were facilitated using a flow cell with a $100 \mathrm{~nm}$ thick diamondlike window, separating the ultrahigh vacuum from the sample gas. Incoming and outgoing radiation passed through the same window, both at an angle of $45^{\circ}$.

The nonresonantly excited x-ray emission spectrum of $\mathrm{N}_{2}$ (Fig. 1, uppermost curve) is in excellent agreement with earlier results $[16,17]$. The spectrum comprises transitions

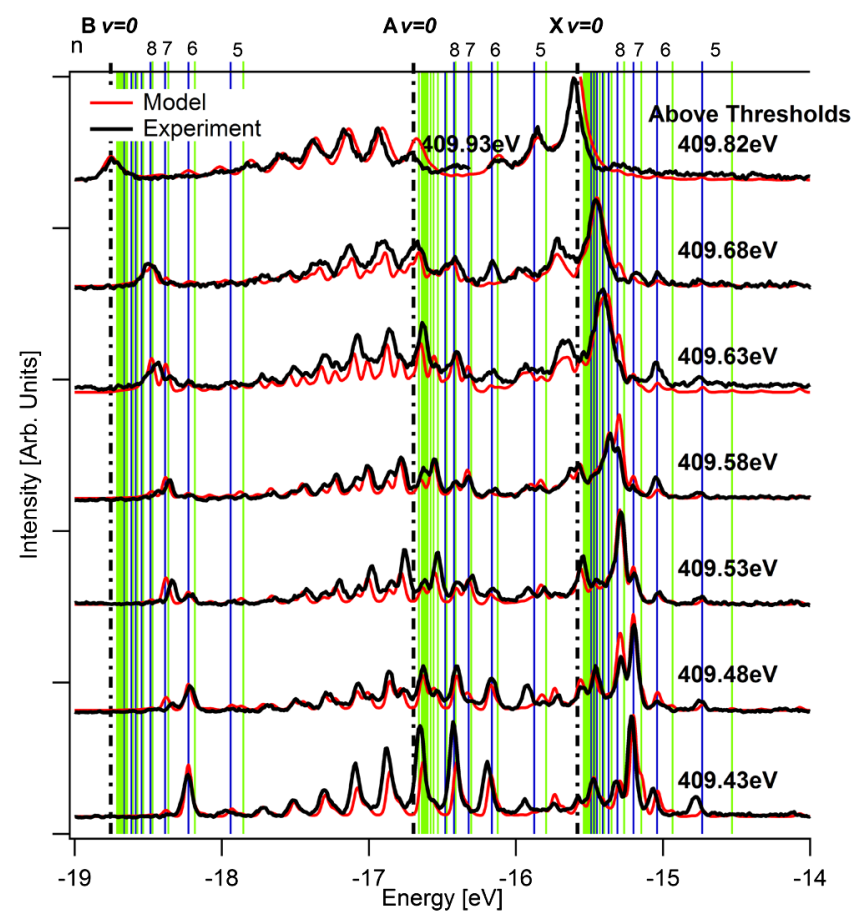

FIG. 1 (color online). RIXS spectra of $\mathrm{N}_{2}$ excited in the vicinity of the core level ionization thresholds (black) compared to the predictions of a simple two-step model (red). Vertical lines show the energies of the $\pi$ (green) and $\sigma$ (blue) states of the Rydberg series converging to the vibrational ground state of the $X$ (15.5808 eV), $A(16.6986 \mathrm{eV})$, and $B(18.7507 \mathrm{eV})$ ionic states. As the overall symmetry of the final states is gerade, the Rydberg electron in the series converging to the gerade $X$ state is gerade, while the Rydberg electron in the series converging to the ungerade $A$ and $B$ states is ungerade. Above the ionization thresholds, the spectra are put on the final state energy scale, using the core-level ionization energies. to the final ionic states which are well separated in energy: $X 3 \sigma_{g}^{-12} \Sigma_{g}^{+}(15.5808 \mathrm{eV}), A 1 \pi_{u}^{-12} \Pi_{u}(16.6986 \mathrm{eV})$, and $B 2 \sigma_{u}^{-1}{ }^{2} \Sigma_{u}^{+}(18.7507 \mathrm{eV})$, where the adiabatic ionization energies are given by photoemission studies [18]. To put the spectrum on the final state (energy loss) scale the emission energy is subtracted from the adiabatic core ionization energies, 409.82 and $409.93 \mathrm{eV}$ for the $\Sigma_{u}$ and $\Sigma_{g}$ states, respectively [9]. We have assumed that the parity selection rule dictated by the dipole approximation is valid, so that ungerade intermediate states populate gerade final states and vice versa. Thus, for the nonresonantly excited spectrum, the peaks assigned to the gerade $X$ final state are aligned to the final state energy scale $0.11 \mathrm{eV}$ differently than the peaks corresponding to ungerade $A$ and $B$ states. A relative $0.11 \mathrm{eV}$ shift would have been easily observed, and the result is clearly in line with strict parity selection: the final states are reached from different intermediate states. The vibrational progressions are dominated by scattering over the vibrational ground state of the core hole states, and the vibrational progressions have been analyzed before $[16,17]$. The dashed-dotted lines in Fig. 1 correspond to the ionization threshold for the vibrational ground states, and ionization thresholds for vibrationally excited valence hole states (not shown) follow at correspondingly higher energy losses. The relative vibrational intensities used throughout the series of spectra are from $v=0$ and up 1.00:0.35:0.20 for the series converging to the $X$ state, 1.00:1.28:1.28:1.06:0.67:0.50:0.33:0.22:0.11:0.06 for the series converging to the $A$ state, and for the series converging to the $B$ threshold only $v=0$ is populated. In the intermediate states, the $v=1$ intensities are set to be $10 \%$ of the $v=0$ intensities. The relative intensities of the states associated with the three valence holes vary slightly through the series of spectra.

Although a steep excitation-energy dependence is observed when the excitation energy is varied in the range from the thresholds down to around $0.5 \mathrm{eV}$ below, the gross spectral shape is largely retained (Fig. 1). This indicates that the coupling between the valence hole and the excited electron is small, as expected for a Rydberg electron. We also note that the spectral features are narrower than the lifetime broadened peaks of the nonresonantly excited spectra, indicating that energetically well-defined final states are populated. On closer inspection, we observe that additional features appear, which have no correspondence in the nonresonantly excited spectrum, and that some peaks show a fine structure with features more closely spaced than the vibrational subpeaks. In the following, we assign the spectra in terms of Rydberg series converging to the thresholds associated with the three lowest ionic states including vibrational excitations.

Already, around $350 \mathrm{meV}$ below the ionization thresholds for $n=6-7$, the energy spacing between adjacent Rydberg levels is smaller than $\Gamma$, which is estimated to be $115 \mathrm{meV}$ [19]. As the thresholds are approached, individual 


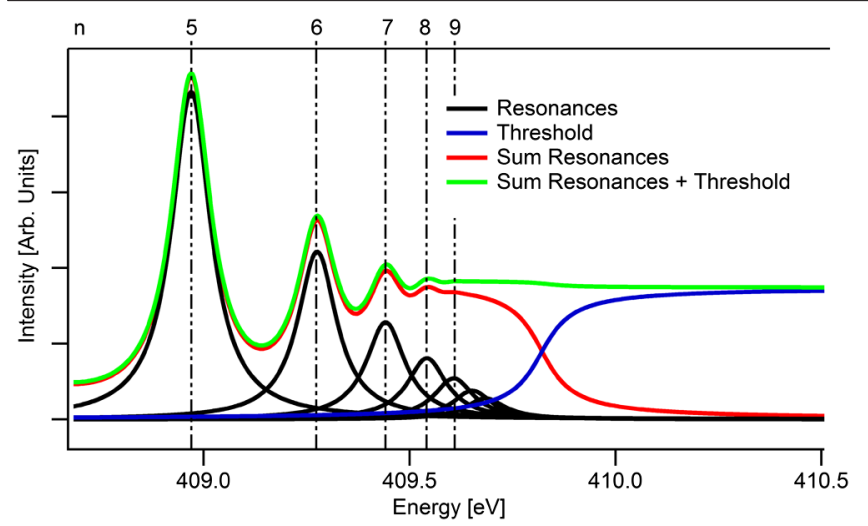

FIG. 2 (color online). As an illustration of the compatibility of the model parameters, the figure shows the smooth onset of the continuum in the absorption spectrum. For clarity, only one series is shown: the $1 \sigma_{u}^{-1} n s \sigma_{g}$ series. The model does not predict the lowenergy peaks properly, as the quantum defects vary at low $n$ [20].

Rydberg states cannot be excited, the absorption spectrum lacks structure, and the flat intensity level in the x-ray absorption spectrum persists so that the onset of the continuum is unobservable (Fig. 2). Below threshold, a superposition of Rydberg states are excited, which, in a time-dependent picture, would correspond to an electronic wave packet which develops during the core-hole lifetime. The radiative decay collapses the wave packet, and welldefined final eigenstates are populated. We assume that the initially excited distribution of Rydberg states is unaffected by the radiative decay, so that the Rydberg electron is a pure spectator during the radiative decay. Then, there is only one channel to each final state, interference effects can be neglected, and a simple quasi-two-step model can be used.

The energy $E_{n}$, of the $n$th Rydberg state in a series converging to an ionization threshold $E_{\mathrm{th}}$ is given by

$$
E_{n}=E_{\mathrm{th}}-\frac{R y}{(n-\delta)^{2}},
$$

where $R y$ is the Rydberg constant and $\delta$ is the quantum defect characterizing the series. The cross section $\sigma_{n}$ for photoexcitation of the Rydberg state with principal quantum number $n$ in a series can be described using the generalized cross section $\tilde{\sigma}$

$$
\sigma_{n}=\frac{\tilde{\sigma}}{(n-\delta)^{3}}
$$

With a well defined energy, $E$, of the incoming photons, the population of the $n$th member of the series, $a_{n}$, depends on the lifetime broadening of the core excited states, $\Gamma$, and $E_{n}$

$$
a_{n} \propto \frac{\tilde{\sigma}}{(n-\delta)^{3}} \frac{\Gamma^{2} / 2}{\left(E-E_{n}\right)^{2}+(\Gamma / 2)^{2}} .
$$

In practice, we must take the finite resolution of the monochromator and spectrometer into account. We multiply by the energy distribution of the monochromator around a nominal excitation energy $E_{\text {in }}$, which we take to be a Gaussian, $G_{\text {in }}\left(E-E_{\text {in }}\right)=e^{-\left(\frac{E-E_{\text {in }}}{2 \sigma_{\text {in }}}\right)^{2}}$, and integrate over all excitation energies to get the population of Rydberg state $n$ at the nominal excitation energy $E_{\text {in }}$.

$$
P\left(E_{\text {in }}, n\right) \propto \int_{E} G_{\text {in }}\left(E-E_{\text {in }}\right) a_{n} d E .
$$

The broadening due to the resolution of the spectrometer is also assumed to be Gaussian, $G_{\text {out }}\left(E_{n}-E_{\text {out }}\right)=$ $e^{-\left(\frac{E_{\text {out }}-E_{n}}{2 \sigma_{\text {out }}}\right)^{2}}$, by which the spectrum is convoluted to get the theoretical spectrum for each nominal energy $E_{\text {in }}$

$$
I\left(E_{\text {in }}, E_{\text {out }}\right) \propto \sum_{n} P\left(E_{\text {in }}, n\right) G_{\text {out }}\left(E_{n}-E_{\text {out }}\right) .
$$

To compare with the measured data, the spectra are finally shifted from the core threshold to the corresponding valence ionization thresholds. The onset of the continua is modeled by a step function broadened by a Lorentzian to account for the lifetime width and normalized to achieve a flat intensity level, and the lifetime-broadened emission following continuum excitation is assumed to appear at threshold. This model is essentially identical to the model presented by Tulkki and Åberg [11], which has earlier been confirmed [21] at high energies without Rydberg resolution.

Apart from experimental functions, the population of all the states in a Rydberg series is, thus, determined by four parameters only: $\tilde{\sigma}, \delta, E_{\mathrm{th}}$, and $\Gamma$, which we take to be constant throughout the series. The approximation that the core-hole lifetime width is independent of the Rydberg electron is valid at high $n$, and $E_{\text {th }}$ is experimentally determined with high accuracy in photoemission and XETECO experiments. Therefore, the entire phenomenology associated with the Rydberg population in one series can be described in terms of two crucial parameters only, $\tilde{\sigma}$ and $\delta$. We have adjusted these parameters for each symmetry-allowed Rydberg series to match the experimental data set (Table I).

The symmetry of the system puts restrictions on the excitations, schematically depicted in Fig. 3. Following dipole selectivity, only gerade Rydberg series converge

\begin{tabular}{|c|c|c|c|c|c|c|}
\hline & \multicolumn{2}{|c|}{$X$ state } & \multicolumn{2}{|c|}{ A state } & \multicolumn{2}{|c|}{$B$ state } \\
\hline & $\mathrm{ns} \sigma$ & $\mathrm{nd} \pi$ & $\mathrm{np} \sigma$ & $\mathrm{np} \pi$ & $\mathrm{np} \sigma$ & $\mathrm{np} \pi$ \\
\hline$\delta$ & 1.00 & 1.40 & 0.90 & 1.10 & 0.90 & 1.10 \\
\hline$\tilde{\sigma}$ & 0.70 & 0.30 & 0.80 & 0.30 & 0.80 & 0.20 \\
\hline
\end{tabular}
to the ungerade core-hole state, and the $1 \sigma_{u}^{-1} n s \sigma_{g}$ and

TABLE I. Values for the quantum defect $(\delta)$ and the generalized cross section $(\tilde{\sigma})$ of the different states that best match the experimental data. 


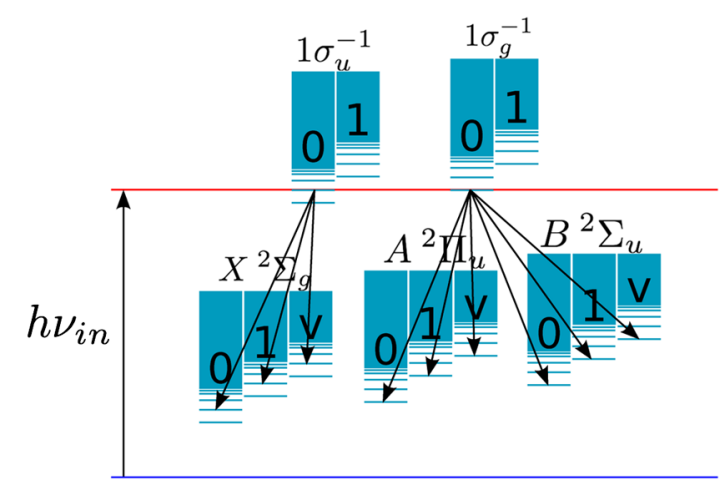

FIG. 3 (color online). Schematic picture of the transitions. A well-defined excitation energy selects Rydberg states just below the $\Sigma_{u}$ and $\Sigma_{g}$ core-level ionization thresholds. The model assumes that the Rydberg population is retained in the transitions to states below the $X, A$, and $B$ thresholds. Symmetry dictates that the core-excited states are ungerade and the final states gerade. Series converging to continua including vibrational excitations are indicated by the $v$ quantum number.

$1 \sigma_{u}^{-1} n d \pi_{g}$ series are the most important (the ungerade or gerade notation for the core orbitals are used for convenience and does not imply that the model regards the core orbitals as delocalized). These channels populate the $3 \sigma_{g}^{-1} n s \sigma_{g}{ }^{1} \Sigma_{g}^{+}$and $3 \sigma_{g}^{-1} n d \pi_{g}{ }^{1} \Pi_{g}$ states, respectively, below the ionic ground state, $X 3 \sigma_{g}^{-1}{ }^{2} \Sigma_{g}^{+}$. The vertical lines in Fig. 1 mark the energies of the $\pi$ (green) and $\sigma$ (blue) states of the series converging to the vibrational ground state, and we see that the states can be readily assigned. At the lowest excitation energy in Fig. 1, the $\pi$ series in the $5 \leq n \leq 8$ range is observed with the maximum intensity at $n=7$. Upon increasing excitation energy, the maximum moves toward higher principal quantum numbers so that the $n=9$ state is most intense at $409.58 \mathrm{eV}$. For higher excitation energies, the maximum is displaced to a region where individual states cannot be resolved, and the shape of the spectrum increasingly mimics the spectrum excited above threshold.

Rydberg series converging to the thresholds of valence excited ions show corresponding behavior. Only ungerade Rydberg series converge to the gerade core hole state, and the most important series are the $1 \sigma_{g}^{-1} n p \sigma_{u}$ and $1 \sigma_{g}^{-1} n p \pi_{u}$ series, populating the $1 \pi_{u}^{-1} n p \sigma_{u}{ }^{1} \Pi_{g}$ and $1 \pi_{u}^{-1} n p \pi_{u}{ }^{1} \Sigma_{g}^{+},{ }^{1} \Sigma_{g}^{-},{ }^{1} \Pi_{g},{ }^{1} \Delta_{g}$ states, respectively, below the ionic $A 3 \pi_{u}^{-12} \Pi_{u}$ state. The model assumes that the coupling between the Rydberg electron and the valence hole is too small to be resolved and it treats the $n p \pi_{u}$ series as a single series. The same intermediate state resonances also populate $2 \sigma_{u}^{-1} n p \sigma_{u}{ }^{1} \Sigma_{g}$ and $2 \sigma_{u}^{-1} n p \pi_{u}{ }^{1} \Pi_{g}$ series converging to the ionic $B 2 \sigma_{u}^{-12} \Pi_{u}$ state. Because of the core-level split, we note that the ungerade and gerade Rydberg resonances are emphasized differently, so that, in the spectrum excited at $409.43 \mathrm{eV}$, the $n=6$ Rydberg state dominates the series converging to the ungerade $A$ and $B$ states where $n=7$ is most intense in the series converging to the $X$ state of the ion.

As indicated in Fig. 3, the series are replicated at thresholds associated with vibrational excitations. The core hole state is dominated by $v=0$ and the model assumes that the relative weights of the vibrational excitations in the final states are the same as in the spectrum excited above thresholds. For the series converging to the $X$ state, replicas up to $v=2$ are observed, whereas Rydberg series converging to vibrational thresholds up to $v=9$ are resolved for the $A$ state.

The agreement between modeled and measured spectra allows for an assignment of virtually all features in the complicated spectrum, and specific Rydberg states with a specific valence and vibrational excitation can be identified. It also gives credibility to the quasi-two-step model, indicating that the threshold dynamics which can be observed at the present level of accuracy is surprisingly simple. The deviation between measured and predicted energy positions of some lower $n$ final states indicates that the single-quantum-defect description of the Rydberg series is inadequate here. In general, this description breaks down at lower $n$ where the Rydberg electron interacts with the electron system in a more complex way. Here, we also expect to find the limitation of the present two-step model. The model relies on the assumption that the Rydberg electron is unaffected by the radiative decay. In this case, no interference effects occur, and the two-step model becomes equivalent with the Kramers-Heisenberg treatment. However, in the case where the emission step does change the Rydberg binding energy, Rydberg shake-up/ down and, thus, electronic-state interference effects would also appear. Then a refined theory is needed, which would have to predict the quantum defect of all series in the intermediate and final states, and to gain predictive power it would most likely have to calculate the energy of each individual state. These energies are not known from experiment, and the quantum defects vary considerably at a lower principal quantum number where Rydberg excitations can be resolved in $\mathrm{x}$-ray absorption spectra [20]. Given more complete information about the involved states, the evaluation of the resonant term of the KramersHeisenberg equation would be straightforward.

The threshold behavior in more complex systems remains to be investigated, and it is obvious that improved resolving power will reveal further fine structure. The method for using RIXS data to construct "absorption spectra with removed lifetime broadening" [12] is now widely applied [1]. Although the construction can be questioned in general [22], it is appropriate insofar as the present quasi-two-step model holds, and absorption features in the immediate vicinity of core thresholds may be observed by high-energy resolution fluorescence detection.

In addition to resolving power, stability and reproducibility will be crucial in future instruments as small energy 
shifts, corresponding to minute mechanical displacements of optical components, have a large impact on the recorded spectra. Here, we have calibrated the absolute energy scale to get the best overall agreement with the model predictions, and we note that allowing for relative shifts in the excitation energy on the order of $10 \mathrm{meV}$ would have further improved the agreement with experiment.

In conclusion, Rydberg-resolved RIXS spectra excited in the intermediate vicinity of core ionization thresholds of $\mathrm{N}_{2}$ are well described within a quasi-two-step model which assumes that the initially excited Rydberg electron is a pure spectator during the final emission. This description implies particularly simple threshold dynamics, and corresponding models can be used whenever a single scattering channel unambiguously populates one final state. This notion improves interpretability of complex spectra and will be helpful as the RIXS technique is further refined and finds application in studies of larger molecules, liquids and solids, and processes therein.

This work was performed at the ADRESS beam line at the Paul Scherrer Institut, Switzerland. The support by the Swedish Research Council (VR) is gratefully acknowledged.

[1] T. Schmitt, F. M. F. de Groot, and J.-E. Rubensson, J. Synchrotron Radiat. 21, 1065 (2014).

[2] M. Simon and T. Schmitt, J. Electron Spectrosc. Relat. Phenom. 188, 1 (2013).

[3] L. J. P. Ament, M. van Veenendaal, T. P. Devereaux, J. P. Hill, and J. van den Brink, Rev. Mod. Phys. 83, 705 (2011).

[4] F. Gel'mukhanov and H. Ågren, Phys. Rep. 312, 87 (1999).

[5] H. Aksela, M. Kivilompolo, E. Nõmmiste, and S. Aksela, Phys. Rev. Lett. 79, 4970 (1997).

[6] J. W. Cooper and R. E. LaVilla, Phys. Rev. Lett. 25, 1745 (1970).
[7] J. J. Bonnet, A. Fleury, M. Bonnefoy, and L. Avan, Phys. Lett. 96A, 13 (1983).

[8] J. Söderström, M. Alagia, R. Richter, S. Stranges, M. Agåker, M. Ström, S. Sorensen, and J.-E. Rubensson, J. Electron Spectrosc. Relat. Phenom. 141, 161 (2004).

[9] M. Alagia, R. Richter, S. Stranges, M. Agå ker, M. Ström, J. Söderström, C. Så the, R. Feifel, S. Sorensen, A. De Fanis et al., Phys. Rev. A 71, 012506 (2005).

[10] J. Söderström, M. Agåker, R. Richter, M. Alagia, S. Stranges, and J.-E. Rubensson, Phys. Rev. A 76, 022505 (2007).

[11] J. Tulkki and T. Åberg, J. Phys. B 15, L435 (1982).

[12] K. Hämäläinen, D. P. Siddons, J. B. Hastings, and L. E. Berman, Phys. Rev. Lett. 67, 2850 (1991).

[13] V. N. Strocov, T. Schmitt, U. Flechsig, T. Schmidt, A. Imhof, Q. Chen, J. Raabe, R. Betemps, D. Zimoch, J. Krempasky et al., J. Synchrotron Radiat. 17, 631 (2010).

[14] G. Ghiringhelli, A. Piazzalunga, C. Dallera, G. Trezzi, L. Braicovich, T. Schmitt, V. N. Strocov, R. Betemps, L. Patthey, X. Wang et al., Rev. Sci. Instrum. 77, 113108 (2006).

[15] J.-E. Rubensson, A. Pietzsch, and F. Hennies, J. Electron Spectrosc. Relat. Phenom. 185, 294 (2012).

[16] L. O. Werme, B. Grennberg, J. Nordgren, C. Nordling, and K. Siegbahn, Nature (London) 242, 453 (1973).

[17] P. Glans, P. Skytt, K. Gunnelin, J.-H. Guo, and J. Nordgren, J. Electron Spectrosc. Relat. Phenom. 82, 193 (1996).

[18] P. Baltzer, M. Larsson, L. Karlsson, B. Wannberg, and M. C. Göthe, Phys. Rev. A 46, 5545 (1992).

[19] K. Prince, M. Vondrek, J. Karvonen, M. Coreno, R. Camilloni, L. Avaldi, and M. de Simone, J. Electron Spectrosc. Relat. Phenom. 101-103, 141 (1999).

[20] J.-I. Adachi, N. Kosugi, and A. Yagishita, J. Phys. B 38, R127 (2005).

[21] M. A. MacDonald, S. H. Southworth, J. C. Levin, A. Henins, R. D. Deslattes, T. LeBrun, Y. Azuma, P. L. Cowan, and B. A. Karlin, Phys. Rev. A 51, 3598 (1995).

[22] P. Carra, M. Fabrizio, and B. T. Thole, Phys. Rev. Lett. 74, 3700 (1995). 